\title{
DHEA Enhances Emotion Regulation Neurocircuits and Modulates Memory for Emotional Stimuli
}

\author{
Rebecca K Sripada*, ${ }^{* 1,2}$, Christine E Marx ${ }^{3,4}$, Anthony P King ${ }^{1,5}$, Nirmala Rajaram ${ }^{1,5}$, Sarah N Garfinkel ${ }^{6}$, \\ James L Abelson' and Israel Liberzon ${ }^{1,2,5}$ \\ 'Department of Psychiatry, University of Michigan, Ann Arbor, MI, USA; '2Department of Psychology, University of Michigan, Ann Arbor, MI, USA; \\ ${ }^{3}$ Department of Psychiatry, Duke University, Durham, NC, USA; ${ }^{4}$ Durham VA Medical Center, Durham, NC, USA; ${ }^{5}$ Ann Arbor VA Medical Center, \\ Ann Arbor, MI, USA; ${ }^{6}$ Department of Psychiatry, Brighton and Sussex Medical School, University of Sussex, Falmer, UK
}

Dehydroepiandrosterone (DHEA) is a neurosteroid with anxiolytic, antidepressant, and antiglucocorticoid properties. It is endogenously released in response to stress, and may reduce negative affect when administered exogenously. Although there have been multiple reports of DHEA's antidepressant and anxiolytic effects, no research to date has examined the neural pathways involved. In particular, brain imaging has not been used to link neurosteroid effects to emotion neurocircuitry. To investigate the brain basis of DHEA's impact on emotion modulation, patients were administered $400 \mathrm{mg}$ of DHEA $(N=14)$ or placebo $(N=15)$ and underwent $3 T$ fMRI while performing the shifted-attention emotion appraisal task (SEAT), a test of emotional processing and regulation. Compared with placebo, DHEA reduced activity in the amygdala and hippocampus, enhanced connectivity between the amygdala and hippocampus, and enhanced activity in the rACC. These activation changes were associated with reduced negative affect. DHEA reduced memory accuracy for emotional stimuli, and also reduced activity in regions associated with conjunctive memory encoding. These results demonstrate that DHEA reduces activity in regions associated with generation of negative emotion and enhances activity in regions linked to regulatory processes. Considering that activity in these regions is altered in mood and anxiety disorders, our results provide initial neuroimaging evidence that DHEA may be useful as a pharmacological intervention for these conditions and invite further investigation into the brain basis of neurosteroid emotion regulatory effects.

Neuropsychopharmacology (2013) 38, 1798-I807; doi:I0.1038/npp.2013.79; published online 24 April 20I3

Keywords: dehydroepiandrosterone; neurosteroid; androsterone; $\mathrm{fMRl}$; emotion regulation

\section{INTRODUCTION}

Dehydroepiandrosterone (DHEA) and its sulfated derivative, DHEA sulfate (DHEAS, hereafter referred to together as DHEA(S)), are cholesterol-derived steroids synthesized in the adrenal glands and de novo in neurons and glia (Baulieu and Robel, 1998). DHEA(S) acts as a GABA(A) receptor noncompetitive antagonist and positive allosteric modulator at the NMDA receptor (for a review, see Maninger et al, 2009). DHEA(S) has neuroprotective, antioxidant, antihypertensive, and anti-inflammatory properties (Maninger et al, 2009), and it reduces conditioned fear responding in rodents (Fleshner et al, 1997) and in humans (Grillon et al, 2006). Owing to this profile of effects, DHEA shows promise as an anxiolytic and antidepressant target in psychiatric disorders.

* Correspondence: Dr RK Sripada, Psychiatry, University of Michigan, 4250 Plymouth Road, 2702 Rachel Upjohn Building, Ann Arbor, Michigan 48109, USA, Tel: + I 7342320190 , Fax: + I 7349367868 , E-mail: rekaufma@umich.edu

Received 25 November 2012; revised 17 February 2013; accepted March 2013; accepted article preview online 3 April 2013
DHEA(S) appears to be dysregulated in mood and anxiety disorders, particularly in major depressive disorder and post traumatic stress disorder (PTSD). Several large-scale studies $(N=700,1147,3000)$ indicate reduced serum DHEA levels in depression (Barrett-Connor et al, 1999; Morsink et al, 2007; Wong et al, 2011). Conversely, numerous studies report normal or elevated DHEA(S) in PTSD (eg, Rasmusson et al, 2004; Spivak et al, 2000), although low levels have also been reported (eg, Kanter et al, 2001). Accumulating evidence suggests that increasing DHEA(S) attenuates anxious and depressive symptomatology, and Rasmusson et al, (2010) have suggested that DHEA elevations in PTSD may represent a compensatory response to stress. In animal models, DHEA(S) administration reduces depressive- and anxiety-like behavior (Melchior and Ritzmann, 1994; Reddy et al, 1998), and three doubleblind, placebo-controlled studies of DHEA administration in major depressive disorder patients reported a significant reduction in depressive symptomatology (Bloch et al, 1999; Schmidt et al, 2005; Wolkowitz et al, 1999). Thus, elevating DHEA(S) levels may be a viable treatment strategy for the reduction of negative affect. In addition to DHEA(S)'s antidepressant effects, numerous studies link DHEA(S) to 
enhancement of memory and executive functioning. In large community samples, serum DHEAS is positively associated with performance in attention, concentration, processing speed, and working memory tasks (Davis et al, 2008; Fonda et al, 2005). No research to date, however, has investigated the mechanism by which DHEA exerts these effects. In particular, no studies have investigated a potential role for DHEA in emotion processing, regulation, and memory for emotional stimuli. This question could be paramount to understanding why DHEA dysregulation is associated with distress and psychopathology.

Growing evidence suggests that DHEA might have a direct impact on emotion neurocircuitry, including the amygdala, hippocampus, insula, and anterior cingulate cortex (ACC). DHEA(S) increases BDNF concentration (Naert et al, 2007) and 5-HT(2A) receptor expression (Cyr et al, 2000) in the amygdala. In humans, a LORETA study indicated that DHEA administration increases activity in the ACC and may impact activity in the hippocampus and insula (Alhaj et al, 2006). DHEAS also modulates dopamine and serotonin release in hippocampal neurons (Zajda et al, 2012). As these four regions are key to emotion regulation and episodic memory (Hofmann et al, 2012), they represent plausible targets through which DHEA could exert its antidepressant effects.

In the current study, we used a probe of emotion induction and modulation/regulation (Klumpp et al, 2011; Sripada et al, 2013) to delineate the brain mechanisms through which DHEA impacts emotional responses and emotion regulation. Given previous behavioral evidence of its anxiolytic and antidepressant effects, we expected DHEA to enhance neural regulatory control of emotions. Given evidence of DHEA dysregulation in stress-related disorders, we further hypothesized it might impact memory for emotional stimuli.

\section{MATERIALS AND METHODS}

\section{Participants}

Study participants were 29 right-handed healthy male volunteers aged 18-34 years (mean $\pm \mathrm{SD}=23 \pm 3.62$ ) recruited from the community via advertisement. Our investigation was restricted to men because baseline levels and metabolism of DHEA vary by gender (Morales et al, 1998), and it was not feasible within the limited scope of this project to recruit enough participants of each gender to analyze each group separately. Exclusion criteria were history of head injury, recent steroid use, and current or past psychiatric disorder, as assessed via the Mini-International Neuropsychiatric Interview (M.I.N.I., Sheehan et al, 1998). Participants were given full details of the study and they provided written informed consent. The study was approved by the Institutional Review Board of the University of Michigan Medical School. All participants completed neurocognitive and self-report measures (see Supplementary Information).

\section{Drug Administration}

Study drug (DHEA) and matching placebo, identical in appearance, were obtained from Belmar Pharmacy
(Lakewood, CO), which provided certificates of analysis. Participants were randomly assigned to receive a single oral dose of $400 \mathrm{mg}$ DHEA $(N=14)$, or placebo $(N=15)$. A dose of $400 \mathrm{mg}$ of DHEA was chosen for this study in order to approach the maximum well-tolerated DHEA dose for which there is currently data available in the clinical scientific literature (Schmidt et al, 2005). In Schmidt et al (2005), a total daily dose of $450 \mathrm{mg}$ per day of DHEA was administered for 3 weeks (following 3 weeks of a lower DHEA dose of $90 \mathrm{mg}$ per day). The choice of $400 \mathrm{mg}$ of DHEA for this study was also informed by a recent pilot investigation in healthy male smokers (http://www.clinicaltrials.gov number NCT00900900), in which a one-time dose of DHEA $400 \mathrm{mg}$ was also utilized. Participants and investigators were blind to condition. DHEA serum concentrations peak 60-480 min after DHEA administration (Arlt et al, 1998). Thus, drug administration occurred $120 \mathrm{~min}$ before neuroimaging to ensure elevated levels during the scan.

\section{Neurosteroid Analyses}

We used circulating levels of DHEA, DHEAS, cortisol, and androsterone as indicators of central neurosteroid levels. Androsterone is a DHEA metabolite that positively modulates $\mathrm{GABA}(\mathrm{A})$ receptors and demonstrates anticonvulsant properties (Kaminski et al, 2005). Serum DHEA levels are closely related to DHEA levels in cerebrospinal fluid (Kancheva et al, 2011), and cerebrospinal fluid DHEA levels are closely related to DHEA levels in brain tissue (Naylor et al, 2008). Serum samples for assay were collected once before drug administration and once after the scanning session, $\sim 3 \mathrm{~h}$ following DHEA administration. Saliva samples were collected four times throughout the experiment-once at baseline, and then once per hour after DHEA administration. Serum DHEA levels were determined via enzyme immunoassay (ALPCO Diagnostics, Salem, NH), and serum and salivary levels of cortisol and DHEAS were determined by chemiluminescent enzyme immunoassay (IMMULITE), according to the manufacturer's directions (Siemens Healthcare Diagnostics, Tarrytown, NY). Androsterone levels in serum were determined by a highly sensitive and specific gas chromatography-mass spectrometry method, as described previously (Marx et al, 2006) with modifications (the electron impact ionization mode was utilized for this investigation rather than negative ion chemical ionization). One microlitre of serum was extracted three times in ethyl acetate before high-performance liquid chromatography purification using tetrahydrofuran, ethanol, and hexane in the mobile phase. All samples were injected in duplicate. All neurosteroid levels were natural log-transformed before analyses.

\section{SEAT Paradigm}

In order to investigate the brain basis of emotional response and regulation, our laboratory has developed an emotion appraisal task (Klumpp et al, 2011; Sripada et al., 2013), modifying the task of Anderson et al (2003). We have previously demonstrated that the SEAT is an effective probe of neurosteroid regulatory effects (Sripada et al, 2013). The SEAT task presents compound stimuli that include both 
emotional faces and neutral scenes (Ekman and Friesen, 1976). Stimuli include composite pictures of superimposed faces (foreground) and buildings (background), as well as 20 pictures of faces or buildings only. The face pictures depict neutral, angry, or fearful expressions, and the building pictures depict indoor or outdoor scenes. In three different conditions, participants are asked to respond to three different questions: (1) 'Gender': whether the face in the foreground is male or female; (2) 'Inside/Outside': whether the scene in the background is indoors or outdoors; or (3) 'Like/Dislike': whether the face in the foreground is liked or disliked. This probes multiple components of emotion regulation, including (1) implicit emotional processing, (2) attentional modulation of emotion, and (3) modulation of emotion by appraisal. The 'Gender' condition probes implicit emotional processing, as attention is focused on an emotional face while one is identifying its gender, and it has been well established that negative emotional faces induce corresponding negative emotions (Dyck et al, 2011; Schneider et al, 1997; Schneider et al, 1994). The 'Inside/Outside' condition engages attentional modulation of emotion, as it requires focusing attention on the building components superimposed on the emotional face. Attention modulation is recognized as a core component of emotion regulation (Gross and Thompson, 2007), and attentional modulation away from threatening faces lowers frustration to subsequent stressful tasks (Johnson, 2009). The 'Like/Dislike' condition engages cognitive appraisal of one's emotional/evaluative state while processing the emotional stimulus. Engaging appraisal has been found to increase activation of dorsal medial prefrontal cortex (Liberzon et al, 2000; Phan et al, 2004), and behavioral studies of emotional appraisal and labeling find that this strategy lowers distress (Lieberman et al, 2011) and facilitates habituation (Tabibnia et al, 2008).

Further details can be found in Supplementary Information.

\section{Memory Task \\ Participants returned $24 \mathrm{~h}$ later for computerized memory testing outside the scanner. Item-specific memory was tested first using yes/no recognition judgments. The conjunctive memory test required participants to make 'match' (previously viewed faces and buildings presented in combinations seen during encoding) or 'mismatch' (items in combinations not previously seen together) judgments (see Garfinkel et al (2013) for details). Participants were encouraged to respond quickly and accurately, but without imposed time limits. Accuracy and reaction time were recorded. Memory sensitivity was determined using signal detection theory, where $\mathrm{d}^{\prime}$ (memory sensitivity) was calculated for conjunctive memory ('match $v s$ mismatch') and item-specific memory ('yes $v s$ no') for faces and buildings. Group effects (DHEA vs placebo) on $\mathrm{d}^{\prime}$ measures (conjunctive, item-specific) were examined using two-tailed independent $t$-tests. The $\mathrm{C}$ (bias) Criterion component of signal detection theory was computed to assess whether participants displayed a response bias (ie, a tendency to endorse or reject items as having been previously pre- sented). In addition, $\mathrm{d}^{\prime}$ values for conjunctive memory were entered as regressors in between-subject analyses.}

\section{Magnetic Resonance Imaging}

Image acquisition. In order to control for potential diurnal variations in DHEA, all scans were conducted in the afternoon. MRI scanning occurred on a Philips 3.0 Tesla Achieva X-series MRI (Philips Medical Systems). After a T1 image (T1-overlay) was obtained, a T2*-weighted, echoplanar acquisition sequence (GRE; repetition time, $2000 \mathrm{~ms}$; echo time, $25 \mathrm{~ms}$; flip angle, $90^{\circ}$; field of view (FOV), $22 \mathrm{~cm}$; 42 slice; thickness/skip, $3.0 / 0.0 \mathrm{~mm}$ matrix size equivalent to $64 \times 64)$ was collected. After discarding three initial volumes to permit thermal equilibration of the MRI signal, 185 volumes were acquired per run. After acquiring the functional volumes, a high-resolution T1 scan was obtained for anatomic normalization (26 FOV; thickness/skip, $1.0 / .00 \mathrm{~mm}$ ). E-prime was used to present stimuli and record responses (Psychology Software Tools, Pittsburgh, $\mathrm{PA}$ ). Participants viewed stimuli through MR-compatible liquid crystal display goggles (NordicNeuroLabs http:// www.nordicneurolab.com) and responded to those stimuli using an MRI-compatible button box.

Preprocessing. A standard series of processing steps was performed using statistical parametric mapping (SPM8; http://www.fil.ion.ucl.ac.uk/spm). Scans were reconstructed, motion-corrected, slice-time corrected, realigned to the first scan in the experiment to correct for head motion, co-registered with the high-resolution sagittal images, anatomically normalized to the Montreal Neurological Institute (MNI) 152 template brain, resampled to $3 \times 3 \times 3 \mathrm{~mm}^{3}$ voxels, and smoothed with an $8 \times 8 \times 8 \mathrm{~mm}^{3}$ kernel. Motion parameters (mean displacement, mean angle) were compared across drug conditions by independent-samples Kruskal-Wallis tests, and runs with any movement $>3 \mathrm{~mm}$ were excluded.

\section{Data Analysis}

Maps of activation in each condition, as well as reaction time and on-line accuracy judgments were analyzed via a 2 (drug type: DHEA or Placebo) $\times 3$ (face type: angry, fearful, neutral) $\times 3$ (condition: male/female, inside/ outside, like/dislike) repeated-measures ANOVA to assess for main effects and interaction effects. Follow-up simple effect analyses were performed with two-tailed $t$-tests, with significance threshold set to 0.05 , corrected for multiple comparisons. As seven different regions of interest (ROIs) were used, the significance threshold was set to $0.05 / 7=0.007$. Levels of delta DHEA and cortisol (endpoint minus baseline) and self-report measures showing significant differences between drug groups were entered as regressors in separate whole-brain analyses, controlling for group membership.

Whole brain and ROI analysis. Z-score images from the individual activation maps were entered into second-level random-effects analyses implemented in SPM8. Secondlevel maps were corrected for multiple comparisons using whole-brain familywise error correction, $p<0.05$. ROI analysis with small volume correction (SVC) was conducted with a priori brain areas identified in previous neuroimaging studies of DHEA (Alhaj et al, 2006). Activation 
threshold and cluster size were determined using AlphaSim (Ward, 2000) to correspond to a false-positive rate of $p<0.05$, corrected for multiple comparisons within ROIs. $A$ priori ROIs of anatomical ACC $(k=819)$, insula $(k=570)$, hippocampus $(k=259)$, and amygdala $(k=63)$ were used as masks. Images were thresholded using a voxelwise threshold of $p<0.005$, uncorrected with a minimum cluster size of 19 connected voxels for ACC, 13 connected voxels for insula, 6 voxels for hippocampus, and 3 voxels for amygdala. The corrected voxelwise probabilities were: ACC $p<0.002$, insula $p<0.002$, hippocampus $p<0.003$, and amygdala $p<0.0036$. Only the activations within the ROIs that survived the volume and voxel correction criteria were extracted and used for further analysis. Unless otherwise stated, all subsequent references to ROIs refer to the clusters within ROIs meeting these requirements. Reported voxel coordinates correspond to standardized MNI space.

The time series from significant clusters within regions of group difference were used in a psychophysiological interaction (PPI) analysis. Deconvolved time series in the anatomical right amygdala was extracted for each participant as the first regressor in the PPI analysis (physiological variable). The second regressor represented the experimental condition (emotion processing; psychological variable). The regressor of interest was the interaction between the time series of the seed region and the experimental condition.

\section{RESULTS}

\section{Participants}

Fourteen participants were administered DHEA and 15 were administered placebo. Groups did not differ by age, ethnicity, self-reported anxiety, or neurocognitive function (in all cases $p>0.1$ ). Sample characteristics, baseline and post-testing neurosteroid levels can be found in Table 1 .

\section{Intervention}

DHEA administration produced 3-4-fold increases in DHEA and DHEAS serum and salivary levels, 4-fold increases in serum androsterone, and did not affect circulating cortisol (see Table 1 and Supplementary Information). Repeated-measures ANOVA (with treatment as a between-subjects factor and DHEA(S) over time as a within-subjects factor) showed that DHEA significantly raised serum DHEAS levels (main effect of treatment: $\mathrm{F}(1,27)=13.70, p<0.001$ and treatment-by-time interaction: $\mathrm{F}(3,81)=15.81, p<0.001$ ), serum DHEA levels (main effect of treatment: $\mathrm{F}(1,27)=4.06, p=0.05$ and treatmentby-time interaction: $\mathrm{F}(1,27)=26.35, p<0.001)$, and salivary DHEAS levels (main effect of treatment: $\mathrm{F}(1,27)=25.20$, $p<0.001$ and treatment-by-time interaction: $\mathrm{F}(1,27)=106.09, \quad p<0.001)$; see Figure 1 . DHEAS was elevated in the DHEA group beginning $60 \mathrm{~min}$ after administration and sustained at 2-3-fold levels throughout

Table I Sample Characteristics and Neurosteroid Levels

\begin{tabular}{|c|c|c|c|c|c|c|}
\hline & \multicolumn{2}{|c|}{ Placebo $(n=15)$} & DHEA $(n=14)$ & \multicolumn{2}{|r|}{$t / \chi^{2}$} & $p$ \\
\hline Age (Mean $\pm \mathrm{SD})$ & \multicolumn{2}{|c|}{$23 \pm 3.0$} & $22 \pm 4.2$ & \multicolumn{2}{|r|}{1.11} & 0.28 \\
\hline Race & \multicolumn{2}{|c|}{$3 \mathrm{~A}, 3 \mathrm{AA}, 9 \mathrm{C}$} & $6 \mathrm{~A}, 8 \mathrm{C}$ & \multicolumn{2}{|c|}{4.03} & 0.13 \\
\hline \multicolumn{7}{|l|}{ Digit span } \\
\hline Baseline & \multicolumn{2}{|c|}{$19.6 \pm 3.9$} & $20.1 \pm 4.6$ & \multicolumn{2}{|r|}{0.30} & 0.77 \\
\hline \multicolumn{7}{|l|}{ Trail-making test } \\
\hline Baseline & \multicolumn{2}{|c|}{$51.4 \pm 15.7$} & $46.9 \pm 10.1$ & \multicolumn{2}{|r|}{0.91} & 0.37 \\
\hline Change & \multicolumn{2}{|c|}{$-7.4 \pm 11.5$} & $-7.6 \pm 5.5$ & \multicolumn{2}{|c|}{0.07} & 0.95 \\
\hline \multicolumn{7}{|l|}{ State trait anxiety inventory } \\
\hline State & \multicolumn{2}{|c|}{$31.7 \pm 7.3$} & $31.7 \pm 5.0$ & \multicolumn{2}{|r|}{0.01} & 0.99 \\
\hline Steroid (Mean \pm SD) & Baseline & Endpoint & Baseline & Endpoint & $t$ & $p$ \\
\hline DHEA (serum; ng/ml) & $28.89 \pm 17.24$ & $25.57 \pm 12.52$ & $24.07 \pm 13.96$ & $80.60 \pm 57.72$ & $3.90 *$ & 0.001 \\
\hline DHEAS (serum; $\mu \mathrm{g} / \mathrm{dl}$ ) & $286.80 \pm|36.9|$ & $313.23 \pm 157.76$ & $284.04 \pm 105.62$ & $1286.68 \pm 481.16$ & 8.03 & $<0.001$ \\
\hline DHEAS (saliva; ng/dl) & $545.84 \pm 486.24$ & $488.57 \pm 303.95$ & $755.61 \pm 1129.57$ & $2700.13 \pm 2410.97$ & 3.91 & 0.001 \\
\hline Androsterone (serum; pg/ml) & $465.50 \pm 204.84$ & $427.88 \pm 189.73$ & $487.76 \pm 152.84$ & $2056.97 \pm 1455.36$ & 4.24 & $<0.001$ \\
\hline Cortisol (serum; $\mu \mathrm{g} / \mathrm{dl}$ ) & $12.79 \pm 4.20$ & $9.24 \pm 2.66$ & $|2.9| \pm 4.19$ & $8.60 \pm 3.48$ & 0.48 & 0.64 \\
\hline Cortisol (saliva; $\mu \mathrm{g} / \mathrm{dl}$ ) & $0.56 \pm 0.24$ & $0.42 \pm 0.34$ & $0.53 \pm 0.24$ & $0.34 \pm 0.13$ & 0.49 & 0.63 \\
\hline
\end{tabular}

Abbreviations: A, Asian; AA, African-American; C, Caucasian; PANAS-X, Positive and Negative Affect Schedule - Expanded Form; VAS, Visual Analogue Scale. Change $=$ endpoint minus baseline.

*t-tests were conducted on change scores (steroid levels at endpoint minus steroid levels at baseline). 

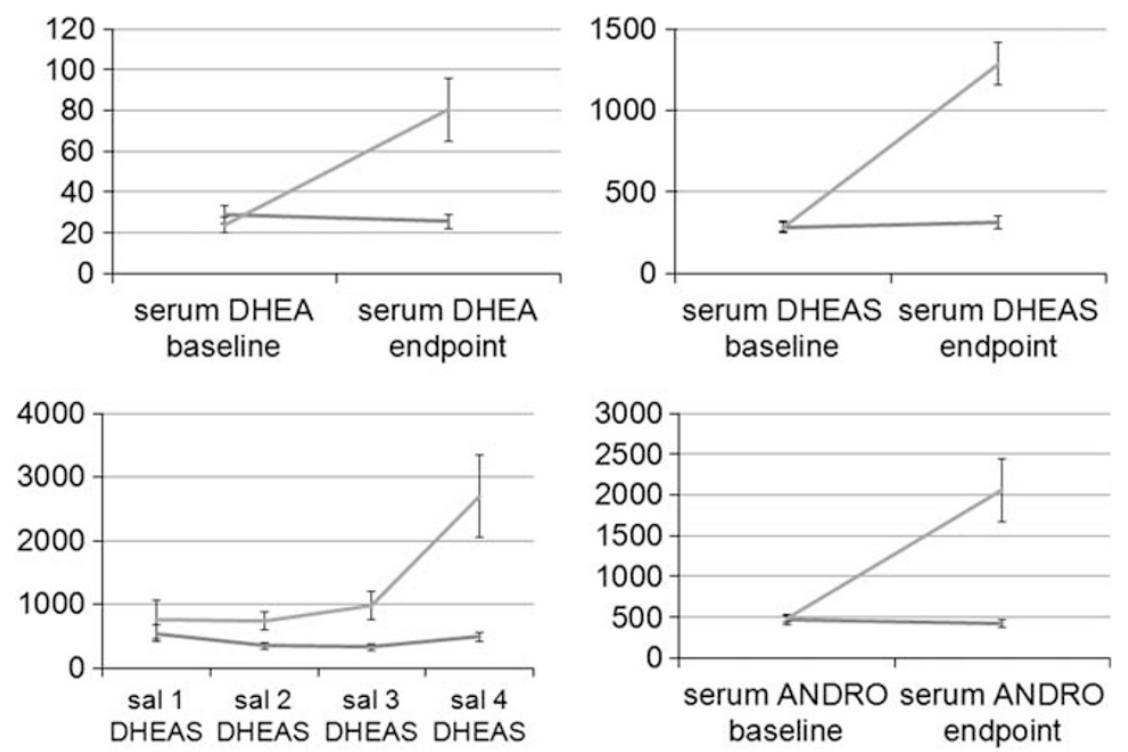

$$
\begin{aligned}
& \text { - PBO } \\
& \text { - DHEA }
\end{aligned}
$$

Figure I As compared with placebo, $400 \mathrm{mg}$ DHEA administration significantly increased serum DHEA (ng/ml), serum DHEAS ( $\mu \mathrm{g} / \mathrm{dl})$, salivary DHEAS $(\mathrm{ng} / \mathrm{dl})$, and serum androsterone $(\mathrm{pg} / \mathrm{ml})$ at $3 \mathrm{~h}$ after administration. Error bars depict SEM. ANDRO, androsterone; PBO, placebo; Sal, salivary.

the course of the experiment in $93 \%$ of participants. There were no significant differences in subjective drug effects $(p>0.4)$. Participants' guesses of which drug they received did not deviate from chance $\left(\chi^{2}(3)=1.81, p=0.61\right)$. Please see Table 1 and Supplementary Information for additional results.

\section{fMRI Results}

Main effect of drug. Compared with placebo, DHEA increased activity in the rostral ACC (rACC), a regulatory region, across all conditions and face types $((3,41,-2)$; $\mathrm{F}=13.30, k=43, p<0.05, \mathrm{SVC}$; see Figure 2). Controlling for group membership, across all conditions and face types, delta serum DHEA level was positively associated with activity in an adjacent region of $\mathrm{rACC}((-6,44,4] ; r=0.692$, $Z=3.23, k=29, p<0.05$, SVC; see Figure 2), indicating that greater peripheral increase in DHEA was associated with greater activation in this regulatory region. Furthermore, within the DHEA group, mean activation in the 43-voxel rACC region of group difference was positively correlated with increase in serum DHEA level $(r=0.633, p=0.015)$. Across groups, mean activation in this same rACC region was also positively correlated with increase in serum androsterone level $(r=0.508, p=0.005)$.

Drug-by-condition interaction. Compared with placebo, DHEA significantly reduced activity in emotion generation regions including the right amygdala/hippocampus $((27,-10,-14) ; \mathrm{F}=6.9, k=10, p<0.05, \mathrm{SVC}$; see Figure 2$)$ and left hippocampus $((-33,-28,-11) ; \mathrm{F}=22.07, k=11$, $p<0.05$, SVC; see Figure 2). Left hippocampus activity was reduced across all conditions and face types $(p<0.001)$, whereas right amygdala/hippocampus activity was reduced specifically in the implicit emotion processing condition (simple effect: $t=2.98, p=0.007$ ). Across all conditions and face types, controlling for group membership, change in serum DHEA level was negatively associated with the right amygdala activity $((36,-4,-14) ; \quad r=-0.672, \quad Z=3.65$, $k=21, p<0.05$, SVC; see Figure 2), indicating that peripheral increase in DHEA was associated with reduced activation in this emotion generation region.

Drug-by-face-type interaction. There were no significant drug-by-face-type interaction effects. Thus, in addition to our three-way (drug $\times$ face type $\times$ condition) ANOVA, we also conducted a two-way (drug $\times$ condition) ANOVA. The results of this additional analysis, which were very similar to the original results, can be found in Supplementary Information.

Subjective effects: Positive and Negative Affect Schedule. In addition, DHEA administration reduced negative affect at trend level, as measured by the Positive and Negative Affect Schedule - Expanded Form (PANAS-X) (PBO: $M=1.36, \mathrm{SD}=0.34 ;$ DHEA $: M=1.18, \mathrm{SD}=0.14 ; t(27)=$ $1.87, p=0.07)$. To examine potential neural underpinnings of this effect, PANAS-X negative affect score was added as a regressor in a separate, exploratory whole-brain analysis. During implicit emotion processing, controlling for group membership, PANAS-X-negative affect score was positively correlated with activity in left hippocampus $((-30$, $-28,-8) ; r=0.573, Z=3.14, k=11 ; p<0.05$, SVC). This finding indicates that decreased activation in left hippocampus is associated with reduced negative emotional response.

Psychophysiological interaction. As right amygdala showed differential between-group activation during implicit emotion processing, subsequent PPI analysis was restricted to the implicit emotion processing condition with 
a
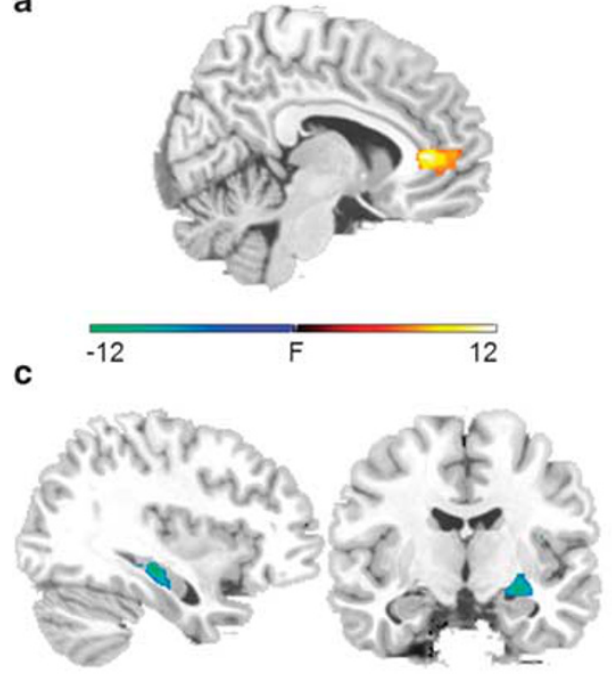
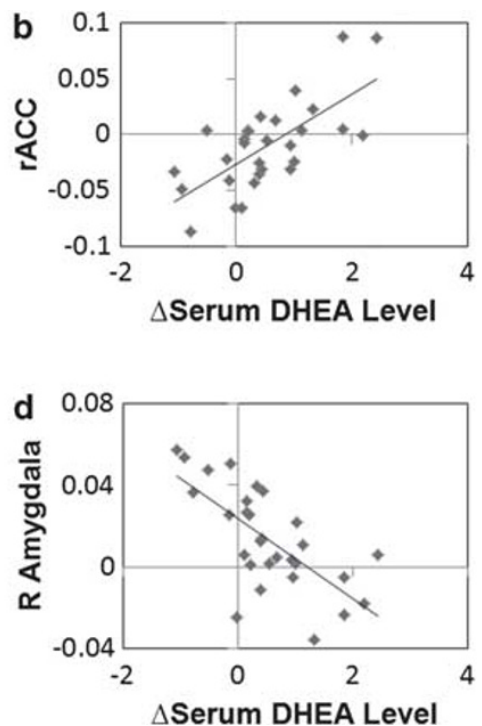

Figure 2 (a) Compared with placebo, DHEA increased rACC activation across conditions and face types $(x=8)$. (b) Serum DHEA predicted increased rACC activation across groups. (c) Compared with placebo, DHEA decreased left hippocampus activation across conditions and face types $(x=-35)$ and decreased right amygdala/hippocampus activation during implicit emotion processing $(y=-9)$. (d) Serum DHEA predicted decreased right amygdala activation across groups.
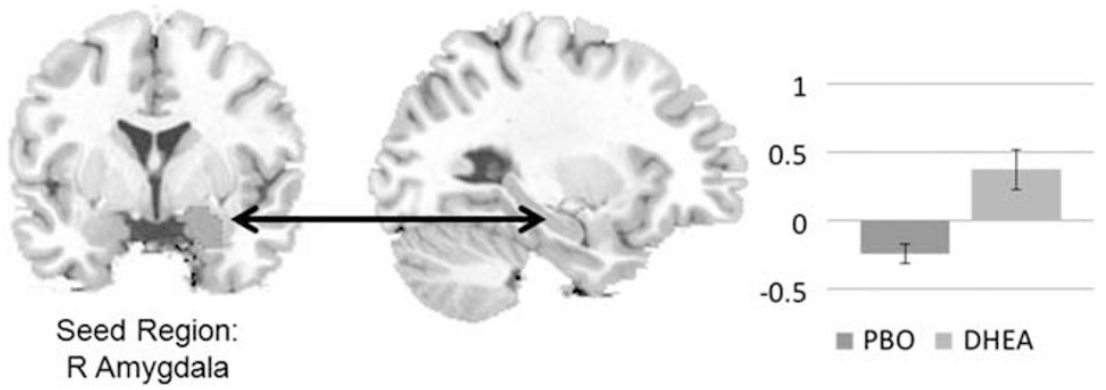

Figure 3 DHEA increased functional connectivity between right amygdala $(y=-18)$ and right hippocampus $(x=28)$ during implicit emotional processing.

anatomical right amygdala as the seed region. Compared with placebo, the DHEA group showed significantly greater functional connectivity between right anatomical amygdala and right anterior hippocampus $((30,-13,-11) ; Z=3.40$, $k=33, p<0.05, S V C)$ and right posterior hippocampus $((24,-40,7) ; Z=3.76, k=14, p<0.05$, SVC; see Figure 3$)$ during the implicit emotion processing condition as compared with implicit baseline. Greater delta serum androsterone level was associated with higher levels of this amygdala-hippocampal connectivity $(r=0.56, p=0.002)$.

\section{Conjunctive memory task}

In the conjunctive memory task, DHEA reduced memory accuracy for emotional stimuli, and also reduced activity in regions associated with conjunctive memory encoding. Conjunctive memory accuracy was reduced in the DHEA group $\left(\mathrm{d}^{\prime} ; t(27)=2.31, p=0.029\right)$ and negatively correlated with serum DHEA across groups $(r=-0.511, p=0.005)$, but DHEA did not impact item-specific memory accuracy or response bias (all $p>0.3$ ). Across all conditions and face types, controlling for group membership, conjunctive memory accuracy was positively correlated with right amygdala $((18,-4,-23) ; \quad r=0.647, \quad Z=3.54, \quad k=22$, $p<0.05$, SVC), left amygdala $((-27,-4,-29) ; r=0.658$, $Z=3.84, k=25, p<0.05, \mathrm{SVC})$, right insula $((39,5,-2)$; $r=0.631, Z=3.61, k=67, p<0.05$, SVC), and left insula activity $\quad((-39,-7,-5) ; \quad r=0.584, \quad Z=3.76, \quad k=58$, $p<0.05$, SVC; see Figure 4$)$, suggesting that activation increases in these regions during encoding predicted successful memory retrieval. Interestingly, endpoint salivary cortisol was positively associated with left hippocampal activity across all conditions and face types $((-24,-31,-5) ; Z=3.44, k=32, p<0.05$, SVC), indicating that greater cortisol level was associated with greater activity in this region.

\section{DISCUSSION}

In this study, we used a probe of emotion induction, modulation, and regulation to assess the neural correlates of DHEA's impact on emotional responses and their regulation. We demonstrated that DHEA reduces activity in regions associated with the generation of negative emotion and enhances activity in regions linked to regulatory control of emotion. These patterns of activation were associated with reduction in self-reported negative affect. In addition, 

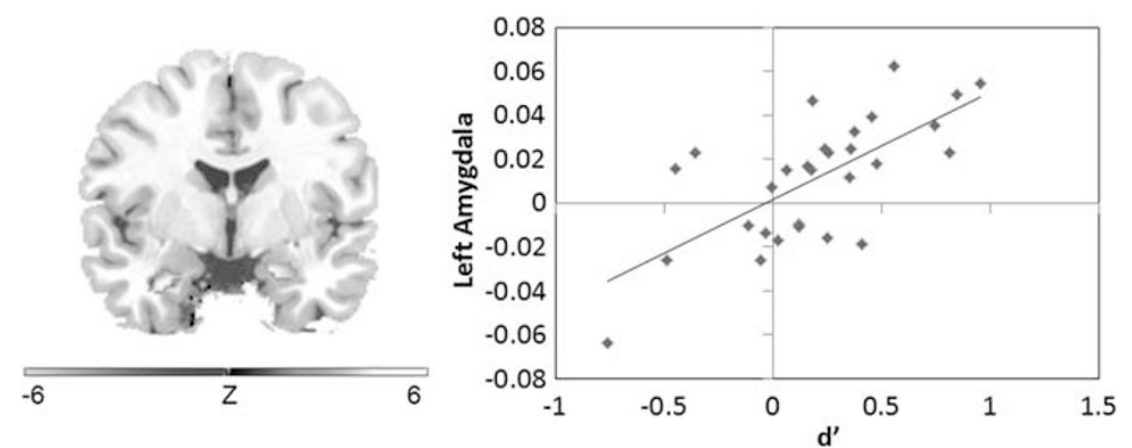

Figure 4 Conjunctive memory accuracy ( $\left.d^{\prime}\right)$ was positively correlated with right hippocampus and bilateral amygdala activation $(y=-2)$.

DHEA reduced conjunctive memory accuracy for emotional stimuli, suggesting a reduction in emotional reactivity during encoding. To our knowledge, this is the first neuroimaging study of acute effects of DHEA administration, and the first to examine DHEA's impact on emotion neurocircuitry. These findings suggest a potential role for DHEA as an anxiolytic and antidepressant agent, and invite further investigation into DHEA enhancement as a novel pharmacological intervention for the treatment of mood and anxiety disorders.

In our sample, single-dose DHEA administration reduced amygdala and hippocampus activity and increased connectivity between these two regions during emotion processing. In addition, the increase in serum DHEA was correlated with decreased amygdala activity, supporting the hypothesis that DHEA reduces emotional reactivity. The amygdala is a key region in emotional salience (Whalen et al, 2001) and the hippocampus is implicated in contextual memory (Maren and Holt, 2000) and fear conditioning (Quirk and Mueller, 2008). Both regions are associated with the detection of threat and production of negative emotional response (Hofmann et al, 2012), and previous research suggests that greater amygdala activation in response to the presentation of facial expressions is associated with greater emotional response (Phan et al, 2004). Thus, DHEA's reduction of activity in amygdala and hippocampus suggests that DHEA may aid in successful downregulation of negative emotions induced by fearful and angry facial expressions, and, by extension, to aversive stimuli in the environment. In support of this notion, DHEA reduced negative affect in our participants, as assessed by the PANAS-X-negative affect subscale (10 items assessing constructs of fear, hostility, guilt, and sadness; Watson and Clark, 1994), albeit at trend level. Our results are consistent with those of large placebo-controlled studies that demonstrated DHEA's antidepressant effects (Bloch et al, 1999; Schmidt et al, 2005; Wolkowitz et al, 1999), and with preclinical studies suggesting that DHEAS is inversely correlated with negative affect (Marx et al, 2006), and that DHEA decreases depressive-like behavior in animals (Reddy et al, 1998). Here, we extend these findings by identifying the neural correlates of this effect.

DHEA administration was also associated with greater rACC activity across conditions and emotions, supporting that DHEA increases activity in regions linked to regulatory control over emotion. Animal studies of fear conditioning implicate mPFC in the extinction of conditioned fear (Milad and Quirk, 2002), and in humans, a recent Granger causality analysis demonstrated an inhibitory influence of the rACC on the amygdala (Zhou et al, 2009). Increased rACC activity and decreased amygdala activity following DHEA administration is thus consistent with the notion of 'top-down' regulation of amygdala by emotional regulatory circuits, a function that is likely diminished in some anxiety disorders (Etkin and Wager, 2007). Postmortem data confirm that DHEA is present in high concentrations in the prefrontal lobe (Lacroix et al, 1987), and one previous neuroimaging study reported that DHEA modulated rACC activity (Alhaj et al, 2006). In our sample, serum increase in both DHEA and androsterone was associated with greater rACC activity, indicating that both neurosteroids may be associated with activity in this region. Androsterone is a metabolite of DHEA which potently modulates $\mathrm{GABA}(\mathrm{A})$ receptors. Owing to its GABAergic activity, androsterone induction represents a potential mechanism for DHEA's moodenhancing effects (Bloch et al, 2006). Increases in rACC activity suggest that DHEA administration may lead to increases in cognitive regulation as well as suppression of negative emotional reactivity. As these capabilities are impaired in some anxiety disorders, our data prompt future investigations into the potential role of DHEA and androsterone as anxiolytic agents.

In addition to modulating activity in brain regions associated with emotion generation and regulation, DHEA also decreased conjunctive memory for emotional stimuli, without affecting other aspects of memory (such as itemspecific recognition). This finding might seem counterintuitive, given previous reports of DHEA's role in attentional enhancement (Ritsner et al, 2006). However, other studies have suggested that DHEA may not benefit memory in healthy individuals (eg, Grimley Evans et al 2006), or might not affect all types of memory in the same way. In our sample, the accuracy of conjunctive memory, specifically conjunctive $\mathrm{d}^{\prime}$, was positively associated with bilateral amygdala activity. This replicates our earlier findings of the neurocircuitry involved in conjunctive memory (Garfinkel et al, 2013). DHEA reduced activity in these regions, suggesting a plausible mechanism by which DHEA might impact associative memory. Specifically, reduced emotional reactivity when viewing the images may have detrimentally affected memory for those images. This is consistent with findings that greater limbic activity in response to greater salience contributes to stronger encoding of emotional stimuli (Murty et al, 2011). 
A reduction in saliency leading to a reduction in emotional memory strength could be relevant to stress-related disorders, such as major depression and PTSD, in which enhanced strength of emotional memories may contribute to pathophysiology. It is important to note that reaction time and accuracy were not impacted by DHEA. Thus, DHEA did not reduce attention to, or impede performance on, the task. Rather, it reduced activity in specific regions related to negative emotion production, and reduced memory for the stimuli. Therefore, DHEA induction may be a plausible mechanism for modulation of emotional reactivity and memory for emotional stimuli. Furthermore, these modulatory effects may be beneficial for individuals with mood and anxiety disorders.

DHEA's reduction of hippocampal activity and its detrimental impact on conjunctive memory may both relate to its role as an antiglucocorticoid. DHEA administration reduces circulating levels of cortisol (Alhaj et al, 2006), and DHEAS is inversely related to peak cortisol in response to $\mathrm{CRH}$ infusion (do Vale et al, 2011). DHEA antagonizes neurotoxic effects of cortisol on the hippocampus (Cardounel et al, 1999), and high DHEA/cortisol ratio in depressed adults is associated with worsened contextual memory (Correa et al, 2012). These findings may indicate a stress-buffering effect of DHEA. In our data, DHEA did not reduce circulating cortisol levels. However, salivary cortisol was positively associated with left hippocampus activity. We have previously reported that cortisol administration increases hippocampal activity during the SEAT task and enhances conjunctive memory (Garfinkel et al, 2013). Thus, DHEA's reduction of hippocampal activity during this task could be associated with the observed decrement in conjunctive memory.

Our finding of reduced memory for emotional stimuli supports the notion that DHEA elevation is a compensatory response to stress. As anxiety disorders and PTSD in particular show consistently elevated levels of DHEA, Rasmusson et al (2010) have suggested that DHEA elevations occur in response to extreme stress, and that greater elevations may be a mark of resiliency. Consistent with this hypothesis, DHEA is positively associated with adaptive responses to stress (Rasmusson et al, 2004) and symptom improvement and coping in PTSD (Yehuda et al, 2010). In addition, 7-keto DHEA reduced PTSD symptoms in a case series of five treatment-refractory patients (Sageman and Brown, 2006). Our findings that DHEA (1) decreased activity of brain regions associated with emotion generation, (2) increased activity in regions linked to effortful and automatic emotion regulation, and (3) reduced associative memory for emotional stimuli, provide novel evidence linking DHEA to processes relevant to resiliency and recovery.

This study has several limitations. First, we measured serum levels of DHEA, and not cerebrospinal fluid or brain levels. However, in animals, these levels are highly correlated (Kancheva et al, 2011). Second, our power to detect differences between groups was limited by our modest sample size; thus, our results should be considered preliminary. Our event-related fMRI design is not well suited to assess DHEA's potential impact on overall brain perfusion and cerebral blood flow. Future studies should employ PET or arterial spin labeling to examine this issue.
Although only conjunctive memory and item-specific memory for emotional faces was measured, it is possible that DHEA could adversely affect normal memory processes. However, previous evidence suggests that higher DHEA is associated with better working memory, episodic memory, and visuospatial memory (Maninger et al, 2009). Thus, it is more likely that our findings represent a specific rather than a general effect. Our sample consisted of healthy male individuals without mood or anxiety disorder diagnoses. Although we report a trend-level improvement of negative affect after DHEA administration, it is a limitation that baseline measures of negative affect were not assessed. Thus, extrapolations to women or to clinical populations should be made with caution. Finally, although we have suggested DHEA as a potential therapeutic approach for anxiety disorders, results from this single dose study should be interpreted cautiously. DHEA's safety and efficacy in modulating emotion neural circuitry beyond a single dose has not been demonstrated.

In conclusion, we demonstrated that DHEA reduces activity in regions associated with the production of negative emotion, enhances activity in regions linked to top-down regulatory control over emotion, and reduces memory accuracy for emotional stimuli. These findings illuminate the neurocircuitry underlying DHEA's effects, and suggest that DHEA may prove useful as a novel pharmacological intervention for the treatment of mood and anxiety disorders.

\section{ACKNOWLEDGEMENTS}

This work is supported by grants from the National Institute of Mental Health (R24 MH075999) to IL, from the Telemedicine and Advanced Technology Research Center (W81XWH-08-2-0208) to IL and AK, from a VA Career Development Transition Award to CM, and by the Veterans Affairs Mid-Atlantic Mental Illness, Research, Education and Clinical Center.

\section{DISCLOSURE}

Dr Marx discloses that she is an applicant or co-applicant on pending US patent applications on the use of neurosteroids and derivatives for the treatment of central nervous system disorders and for lowering cholesterol (no patents issued, no licensing in place), and she is an unpaid scientific advisor to Sage Therapeutics. The remaining authors declare no conflict of interest.

\section{REFERENCES}

Alhaj HA, Massey AE, McAllister-Williams RH (2006). Effects of DHEA administration on episodic memory, cortisol and mood in healthy young men: a double-blind, placebo-controlled study. Psychopharmacology (Berl) 188: 541-551.

Anderson AK, Christoff K, Panitz D, De Rosa E, Gabrieli JD (2003). Neural correlates of the automatic processing of threat facial signals. J Neurosci 23: 5627-5633.

Arlt W, Justl HG, Callies F, Reincke M, Hubler D, Oettel M et al (1998). Oral dehydroepiandrosterone for adrenal androgen replacement: pharmacokinetics and peripheral conversion to androgens and estrogens in young healthy females after 
dexamethasone suppression. J Clin Endocrinol Metab 83: 1928-1934.

Barrett-Connor E, von Muhlen D, Laughlin GA, Kripke A (1999). Endogenous levels of dehydroepiandrosterone sulfate, but not other sex hormones, are associated with depressed mood in older women: the Rancho Bernardo Study. J Am Geriatr Soc 47: 685-691.

Baulieu EE, Robel P (1998). Dehydroepiandrosterone (DHEA) and dehydroepiandrosterone sulfate (DHEAS) as neuroactive neurosteroids. Proc Natl Acad Sci USA 95: 4089-4091.

Bloch M, Rubinow DR, Berlin K, Kevala KR, Kim HY, Schmidt PJ (2006). Monoamines and neurosteroids in sexual function during induced hypogonadism in healthy men. Arch Gen Psychiatry 63: 450-456.

Bloch M, Schmidt PJ, Danaceau MA, Adams LF, Rubinow DR (1999). Dehydroepiandrosterone treatment of midlife dysthymia. Biol Psychiatry 45: 1533-1541.

Cardounel A, Regelson W, Kalimi M (1999). Dehydroepiandrosterone protects hippocampal neurons against neurotoxininduced cell death: mechanism of action. Proc Soc Exp Biol Med 222: 145-149.

Correa MS, Balardin JB, Caldieraro MA, Fleck MP, Argimon I, Luz $C$ et al (2012). Contextual recognition memory deficits in major depression are suppressed by cognitive support at encoding. Biol Psychol 89: 293-299.

Cyr M, Landry M, Di Paolo T (2000). Modulation by estrogenreceptor directed drugs of 5-hydroxytryptamine-2A receptors in rat brain. Neuropsychopharmacology 23: 69-78.

Davis SR, Shah SM, McKenzie DP, Kulkarni J, Davison SL, Bell RJ (2008). Dehydroepiandrosterone sulfate levels are associated with more favorable cognitive function in women. J Clin Endocrinol Metab 93: 801-808.

do Vale S, Martin Martins J, Fagundes MJ, do Carmo I (2011). Plasma dehydroepiandrosterone-sulphate is related to personality and stress response. Neuro Endocrinol Lett 32: 442-448.

Dyck M, Loughead J, Kellermann T, Boers F, Gur RC, Mathiak K (2011). Cognitive versus automatic mechanisms of mood induction differentially activate left and right amygdala. NeuroImage 54: 2503-2513.

Ekman P, Friesen WV (1976). Pictures of Facial Affect. Consulting Psychologists Press: Palo Alto, CA.

Etkin A, Wager TD (2007). Functional neuroimaging of anxiety: a meta-analysis of emotional processing in PTSD, social anxiety disorder, and specific phobia. Am J Psychiatry 164: 1476-1488.

Fleshner M, Pugh CR, Tremblay D, Rudy JW (1997). DHEA-S selectively impairs contextual-fear conditioning: support for the antiglucocorticoid hypothesis. Behav Neurosci 111: 512-517.

Fonda SJ, Bertrand R, O’Donnell A, Longcope C, McKinlay JB (2005). Age, hormones, and cognitive functioning among middle-aged and elderly men: cross-sectional evidence from the Massachusetts male aging study. J Gerontol A Biol Sci Med Sci 60: 385-390.

Garfinkel SN, Ho SS, Wang X, Abelson JL, Taylor SF, Gonzalez R et al (2013). Cortisol facilitates memory by enhancing hippocampal activation and functional connectivity. under review.

Grillon C, Pine DS, Baas JM, Lawley M, Ellis V, Charney DS (2006). Cortisol and DHEA-S are associated with startle potentiation during aversive conditioning in humans. Psychopharmacology (Berl) 186: 434-441.

Grimley Evans J, Malouf R, Huppert FAH, van Niekerk JK (2006). Dehydroepiandrosterone (DHEA) supplementation for cognitive function in healthy elderly people. Cochrane Database Syst Rev Art No: CD006221.

Gross JJ, Thompson RA (2007). Emotion regulation conceptual foundations. In: Gross JJ (ed) Handbook of emotion regulation. Guillford Press: New York, pp 3-24.

Hofmann SG, Ellard KK, Siegle GJ (2012). Neurobiological correlates of cognitions in fear and anxiety: a cognitive-neurobiological information-processing model. Cogn Emot 26: 282-299.

Johnson DR (2009). Goal-directed attentional deployment to emotional faces and individual differences in emotional regulation. J Res Pers 43: 8-13.

Kaminski RM, Marini H, Kim WJ, Rogawski MA (2005). Anticonvulsant activity of androsterone and etiocholanolone. Epilepsia 46: 819-827.

Kancheva R, Hill M, Novak Z, Chrastina J, Kancheva L, Starka L (2011). Neuroactive steroids in periphery and cerebrospinal fluid. Neuroscience 191: 22-27.

Kanter ED, Wilkinson CW, Radant AD, Petrie EC, Dobie DJ, McFall ME et al (2001). Glucocorticoid feedback sensitivity and adrenocortical responsiveness in posttraumatic stress disorder. Biol Psychiatry 50: 238-245.

Klumpp H, Ho SS, Taylor SF, Phan KL, Abelson JL, Liberzon I (2011). Trait anxiety modulates anterior cingulate activation to threat interference. Depress Anxiety 28: 194-201.

Lacroix C, Fiet J, Benais JP, Gueux B, Bonete R, Villette JM et al (1987). Simultaneous radioimmunoassay of progesterone, androst-4-enedione, pregnenolone, dehydroepiandrosterone and 17-hydroxyprogesterone in specific regions of human brain. J Steroid Biochem 28: 317-325.

Liberzon I, Taylor SF, Fig LM, Decker LR, Koeppe RA, Minoshima S (2000). Limbic activation and psychophysiologic responses to aversive visual stimuli. Interaction with cognitive task. Neuropsychopharmacology 23: 508-516.

Lieberman MD, Inagaki TK, Tabibnia G, Crockett MJ (2011). Subjective responses to emotional stimuli during labeling, reappraisal, and distraction. Emotion 11: 468-480.

Maninger N, Wolkowitz OM, Reus VI, Epel ES, Mellon SH (2009). Neurobiological and neuropsychiatric effects of dehydroepiandrosterone (DHEA) and DHEA sulfate (DHEAS). Front Neuroendocrinol 30: 65-91.

Maren S, Holt W (2000). The hippocampus and contextual memory retrieval in Pavlovian conditioning. Behav Brain Res 110: $97-108$.

Marx CE, Trost WT, Shampine L, Behm FM, Giordano LA, Massing MW et al (2006). Neuroactive steroids, negative affect, and nicotine dependence severity in male smokers. Psychopharmacology (Berl) 186: 462-472.

Melchior CL, Ritzmann RF (1994). Dehydroepiandrosterone is an anxiolytic in mice on the plus maze. Pharmacol Biochem Behav 47: 437-441.

Milad MR, Quirk GJ (2002). Neurons in medial prefrontal cortex signal memory for fear extinction. Nature 420: 70-74.

Morales AJ, Haubrich RH, Hwang JY, Asakura H, Yen SS (1998). The effect of six months treatment with a $100 \mathrm{mg}$ daily dose of dehydroepiandrosterone (DHEA) on circulating sex steroids, body composition and muscle strength in age-advanced men and women. Clin Endocrinol (Oxf) 49: 421-432.

Morsink LF, Vogelzangs N, Nicklas BJ, Beekman AT, Satterfield S, Rubin SM et al (2007). Associations between sex steroid hormone levels and depressive symptoms in elderly men and women: results from the Health ABC study. Psychoneuroendocrinology 32: 874-883.

Murty VP, Ritchey M, Adcock RA, LaBar KS (2011). Reprint of: fMRI studies of successful emotional memory encoding: a quantitative meta-analysis. Neuropsychologia 49: 695-705.

Naert G, Maurice T, Tapia-Arancibia L, Givalois L (2007). Neuroactive steroids modulate HPA axis activity and cerebral brain-derived neurotrophic factor (BDNF) protein levels in adult male rats. Psychoneuroendocrinology 32: 1062-1078.

Naylor JC, Hulette CM, Steffens DC, Shampine LJ, Ervin JF, Payne VM et al (2008). Cerebrospinal fluid dehydroepiandrosterone levels are correlated with brain dehydroepiandrosterone levels, elevated in Alzheimer's disease, and related to neuropathological disease stage. J Clin Endocrinol Metab 93: 3173-3178. 
Phan KL, Taylor SF, Welsh RC, Ho SH, Britton JC, Liberzon I (2004). Neural correlates of individual ratings of emotional salience: a trial-related fMRI study. NeuroImage 21: 768-780.

Quirk GJ, Mueller D (2008). Neural mechanisms of extinction learning and retrieval. Neuropsychopharmacology 33: 56-72.

Rasmusson AM, Schnurr PP, Zukowska Z, Scioli E, Forman DE (2010). Adaptation to extreme stress: post-traumatic stress disorder, neuropeptide $\mathrm{Y}$ and metabolic syndrome. Exp Biol Med (Maywood) 235: 1150-1162.

Rasmusson AM, Vasek J, Lipschitz DS, Vojvoda D, Mustone ME, Shi $Q$ et al (2004). An increased capacity for adrenal DHEA release is associated with decreased avoidance and negative mood symptoms in women with PTSD. Neuropsychopharmacology 29: $1546-1557$.

Reddy DS, Kaur G, Kulkarni SK (1998). Sigma (sigma1) receptor mediated anti-depressant-like effects of neurosteroids in the Porsolt forced swim test. Neuroreport 9: 3069-3073.

Ritsner MS, Gibel A, Ratner Y, Tsinovoy G, Strous RD (2006). Improvement of sustained attention and visual and movement skills, but not clinical symptoms, after dehydroepiandrosterone augmentation in schizophrenia: a randomized, double-blind, placebo-controlled, crossover trial. J Clin Psychopharmacol 26: 495-499.

Sageman S, Brown RP (2006). 3-acetyl-7-oxo-dehydroepiandrosterone for healing treatment-resistant posttraumatic stress disorder in women: 5 case reports. J Clin Psychiatry 67: 493-496.

Schmidt PJ, Daly RC, Bloch M, Smith MJ, Danaceau MA St, Clair LS et al (2005). Dehydroepiandrosterone monotherapy in midlife-onset major and minor depression. Arch Gen Psychiatry 62: $154-162$.

Schneider F, Grodd W, Weiss U, Klose U, Mayer KR, Nagele T et al (1997). Functional MRI reveals left amygdala activation during emotion. Psychiatry Res 76: 75-82.

Schneider F, Gur RC, Gur RE, Muenz LR (1994). Standardized mood induction with happy and sad facial expressions. Psychiatry Res 51: 19-31.

Sheehan DV, Lecrubier Y, Sheehan KH, Amorim P, Janavs J, Weiller E et al (1998). The Mini-International Neuropsychiatric Interview (M.I.N.I.): the development and validation of a structured diagnostic psychiatric interview for DSM-IV and ICD-10. J Clin Psychiatry 59, Suppl 20, 22-33, quiz 34-57.

Spivak B, Maayan R, Kotler M, Mester R, Gil-Ad I, Shtaif B et al (2000). Elevated circulatory level of GABA(A): antagonistic neurosteroids in patients with combat-related post-traumatic stress disorder. Psychol Med 30: 1227-1231.

Sripada RK, Marx CE, King AP, Rampton JC, Ho SH, Liberzon I (2013). Allopregnanolone Elevations Following Pregnenolone Administration are Associated with Enhanced Activation of Emotion Regulation Neurocircuits. Biol Psychiatry S0006-3223: 01093-1.

Tabibnia G, Lieberman MD, Craske MG (2008). The lasting effect of words on feelings: words may facilitate exposure effects to threatening images. Emotion 8: 307-317.

Ward B (2000): Simultaneous inference for fMRI data http:// afni.nimh.nih.gov/pub/dist/doc/manual/AlphaSim.pdf.

Watson D, Clark LA (1994). Manual for the Positive and Negative Affect Schedule-Expanded Form. University of Iowa: Iowa City, IA.

Whalen PJ, Shin LM, McInerney SC, Fischer H, Wright CI, Rauch SL (2001). A functional MRI study of human amygdala responses to facial expressions of fear versus anger. Emotion 1: 70-83.

Wolkowitz OM, Reus VI, Keebler A, Nelson N, Friedland M, Brizendine L et al (1999). Double-blind treatment of major depression with dehydroepiandrosterone. Am J Psychiatry 156: 646-649.

Wong SY, Leung JC, Kwok T, Ohlsson C, Vandenput L, Leung PC et al (2011). Low DHEAS levels are associated with depressive symptoms in elderly Chinese men: results from a large study. Asian J Androl 13: 898-902.

Yehuda R, Bierer LM, Pratchett LC, Pelcovitz M (2010). Using biological markers to inform a clinically meaningful treatment response. Ann N Y Acad Sci 1208: 158-163.

Zajda ME, Krzascik P, Hill M, Majewska MD (2012). Psychomotor and rewarding properties of the neurosteroids dehydroepiandrosterone sulphate and androsterone: effects on monoamine and steroid metabolism. Acta Neurobiol Exp (Wars) 72: 65-79.

Zhou Z, Ding M, Chen Y, Wright P, Lu Z, Liu Y (2009). Detecting directional influence in fMRI connectivity analysis using PCA based Granger causality. Brain Res 1289: 22-29.

Supplementary Information accompanies the paper on the Neuropsychopharmacology website (http://www.nature.com/npp) 